\title{
ETM - An Optimized Routing Protocol for Mobile SSM Sources
}

\author{
Thomas C. Schmidt, Matthias Wahliisch and Maik Wodarz
}

\author{
Thomas C. Schmidt, Maik Wodarz \\ HAW Hamburg, Dept. Informatik, Berliner Tor 7, 20099 Hamburg, Germany e-mail: \\ t.schmidt@ieee.org, Maik.Wodarz@informatik.haw-hamburg.de \\ Matthias Wählisch \\ link-lab, Hönower Str. 35, 10318 Berlin, Germany e-mail: waehlisch@ieee.org \\ He is also with HAW Hamburg, Dept. Informatik
}

\begin{abstract}
Mobility is considered a key technology of the next generation Internet and has been standardized within the IETF. Rapidly emerging multimedia group applications such as IPTV, MMORPGs and video conferencing increase the demand for mobile group communication, but a standard design of mobile multicast is still awaited. The traditional Internet approach of Any Source Multicast (ASM) routing remains hesitant to spread beyond walled gardens, while the simpler and more selective Source Specific Multicast (SSM) is expected to globally disseminate to many users and services. However, mobility support for Source Specific Multicast is still known to be a major open problem. In this paper we introduce the Enhanced Tree Morphing (ETM), an optimized multicast routing protocol for transforming (morphing) source specific distribution trees into optimal trees rooted at a relocated source. Following up on previous results for the initial Tree Morphing, we present enhancements that lead to a simpler protocol with significantly optimized performance values in both, forwarding performance and protocol robustness. Extensive evaluations based on real-world topology data are part of this work.
\end{abstract}

\section{Introduction}

Mobile communication today follows the trend to converge with networked applications to a common IP-centric world. Throughout this process, Internet services are expected to extend to mobility management in the near future. The virginal availability of a new, truly mobile IP enabled network layer [9] offers connectivity to nomadic users at roaming devices, while preserving communication sessions beyond IP subnet changes. Voice and video (group) conferencing, as well as large scale content distribution (e.g., IPTV) and massive multiplayer games (MMORPGs) are considered the key applications for the next generation ubiquitous Internet. Inexpensive, point-to-multipoint enabled technologies such as IEEE 802.16 or DVB-H/ IPDC emerge on the subnetwork layer and facilitate large-scale group communi- 
cation deployment. But unlike point-to-point mobility and despite of ten research years, mobile multicast protocol development is still in a premature state [17].

This paper addresses the issue of mobile Source Specific Multicast routing on the network layer in presenting the Enhanced Tree Morphing protocol (ETM). Source Specific Multicast (SSM) [7], just released as an initial standard, is considered a deployment-friendly variant of group distribution techniques. In contrast to Any Source Multicast (ASM) [3], optimal (S,G) multicast source trees are constructed immediately from $(S, G)$ subscriptions at the client side, without utilizing network flooding or rendezvous points. Source addresses are to be acquired out of band, which a SIP [13] session initiation in conferencing scenarios may facilitate [16].

Conferencing parties request seamless real-time performance of a mobility aware group communication service, thereby attaining the simultaneous roles of mobile multicast listener and source. We discuss session mobility in the context of real-time multicast communication and present an optimized protocol, which adapts to sender mobility with minimal impact on service quality. The Tree Morphing protocol (TM) $[14,15]$, one of the few approaches to SSM source mobility management, was designed to enable immediate, unencapsulated multicast data transmission subsequent to Mobile IPv6 handovers. Nevertheless, a previously undertaken extensive analysis of the early scheme revealed shortcomings and opportunities for improvement.

As will be shown in the remaining paper, ETM overcomes all issues of the TM and adheres to real-time compliant performance in various, realistic routing topologies. The simplified scheme can be implemented with little overhead, and by applying minimal modifications to current standard protocols for unicast mobility man-

agement. In this paper we first discuss the mobile multimedia group conferencing problem and related work. In section 3 we present the core protocol. A thorough evaluation follows in section 4. Finally, section 5 is dedicated to a conclusion and an outlook.

\section{The Mobile Source Specific Multicast Problem and Related Work}

\subsection{Problem Statement}

A mobile multicast sender will face the problem of enabling a continuous forwarding of data to its group of receivers, while it undergoes roaming and network layer handovers. Its mobility protocol should facilitate a seamless transmission service and at the same time preserve transparency with respect to network and address changes at the receiver side. Multicast listener applications are frequently source address aware. A mobile multicast source consequently must meet address transparency at two layers: To comply with RPF checks, it has to use an address within the IPv6 basic header's source field, which is in topological concordance with the employed multicast distribution tree. For application transparency, the logical node 
identifier, commonly the Home Address, must be presented as the packet source address to the transport layer at the receivers.

At the complementary side, network routing must comply with the sender movement without having network functionality compromised. It should realize native forwarding whenever possible to preserve its resources, but needs to ensure routing convergence even under a rapid movement of the sender. Mobility support for multicast sources at the network layer thus poses a significant challenge to the infrastructure. An SSM node submitting data to a group of receivers defines the root of a source specific shortest path tree (SPT), distributing data towards its receivers. Native forwarding along source specific delivery trees will be bound to the source's topological network address due to reverse path forwarding (RPF) checks. A mobile multicast source moving to a new subnetwork is only able to either inject data into a previously established delivery tree, which may be a rendezvous point based shared tree, or to (re-)initiate the construction of a multicast distribution tree compliant to its new location. In the latter case, the mobile sender will have to precede without controlling the new tree development, as it operates decoupled from its receivers.

\subsection{Related Work}

Three principal approaches to SSM source mobility are presently around.

\section{Statically Rooted Distribution Trees}

The MIPv6 standard proposes bi-directional tunneling through the home agent as a minimal multicast support for mobile senders and listeners as introduced by [21]. In this approach, the mobile multicast source (MS) always uses its Home Address (HoA) for multicast operations. Static home agents hide mobility completely from multicast routing at the price of triangular paths and extensive encapsulation.

Following a shared tree approach, [12] propose to employ Rendezvous Points of PIM-SM [4] as mobility anchors. Mobile senders tunnel their data to these "Mobility-aware Rendezvous Points" (MRPs), whence in restriction to a single domain this scheme is equivalent to the bi-directional tunneling. Focusing on interdomain mobile multicast, the authors design a tunnel- or SSM-based backbone distribution of packets between MRPs.

\section{Reconstruction of Distribution Trees}

Several authors propose to construct a completely new distribution tree after the movement of a mobile source. These schemes have to rely on client notification for initiating new router state establishment. At the same time they need to preserve address transparency to the client. To account for the latter, Thaler [18] proposes to employ binding caches and to obtain source address transparency analogous to MIPv6 unicast communication. Initial session announcements and changes of source addresses are to be distributed periodically to clients via an additional multicast control tree based at the home agent. Source-tree handovers are then activated on listener requests. Jelger and Noel [8] suggest handover improvements by 
employing anchor points within the source network, supporting a continuous data reception during client-initiated handovers.

\section{Tree Modification Schemes}

Very little attention has been given to procedures, which modify existing distribution trees to continuously serve for data transmission of mobile sources. In the ASM case of DVMRP routing, [2] propose an algorithm to extend the root of a given delivery tree to incorporate a new source location. O'Neill [10] suggests a scheme to overcome RPF-check failures originating from multicast source address changes, by introducing an extended routing information, which accompanies data in a Hop-by-Hop option header.

A routing protocol adaptive to SSM source mobility, the Tree Morphing, has been introduced by the authors in [14]. A mobile multicast source (MS) away from home will transmit unencapsulated data to a group, using its current CoA on the Internet layer, but HoA on the application layer, which is carried in extension headers like in MIPv6. In extension to unicast routing, though, the entire Internet layer, i.e. routers included, will be aware of the permanent HoA. Maintaining binding-cachelike address pairs in router states will enable all routers to simultaneously identify $(H o A, G)$-based group membership and $(C o A, G)$-based tree topology. When moving to a new point of attachment, the MS will alter its address from previous CoA (pCoA) to new CoA (nCoA) and eventually change from its previous Designated multicast Router (pDR) to a next Designated Router (nDR). Subsequent to handover it will immediately continue to deliver data along an extension of its previous source tree using source routing from nDR to pDR. All routers along this path will learn the new CoA of MS and implement appropriate forwarding states.

Routers on this extended tree use RPF checks to discover potential short cuts. In the absence of short cuts, routers re-use those parts of the previous delivery tree, which coincide with the new shortest path tree. Only branches of the new shortest path tree, which have not previously been established, need to be constructed. In this way, the previous shortest path tree will be morphed into a next shortest path tree.

\subsection{Discussion}

Bi-directional tunneling is considered only a minimal solution, operational without explicit multicast mobility support. It introduces considerable overheads from triangular routing and wide spanned encapsulation. Handover assistance via mobilityaware rendezvous points relies on triangular routing and tunneling, as well, and in addition re-introduces RPs, which are not native to SSM routing.

Receiver oriented tree reconstructions in SSM suffer from the problem of receivers being unsynchronized to source handovers. The adoption of a persistent control tree will resolve this issue, but introduces the overhead of an additional multicast tree spanning all receivers. In addition, handover messages distributed via a control tree will require a roundtrip signaling between source and receivers and consequently may be slow in widespread topologies. The MSSMSv6 approach [8] will 
expedite the handover process, but fails to solve client synchronization. The authors henceforth are leaving the source in case of its rapid movement with an unlimited number of 'historic' delivery trees to be fed simultaneously.

Adaptive tree approaches offer a solution space that addresses both, seamless handovers and sustained receiver contact. In addition, they can take advantage of limited mobility-related changes in the shapes of multicast distribution trees that can be observed [20]. The Tree Morphing protocol complies well with these advantages, but suffers from two significant drawbacks. At first, it relies on an initial source routing of packets, which appears elegant from the perspective of protocol design, but faces severe deployment objections from the perspective of operators. Source routing is considered a general security threat and frequently defeated. At second, extensive simulation studies revealed not only an undesirable delay stretch in initial packet delivery, but a realistic likelihood that the router convergence process will cause packet loss for those packets, initially routed via the previous designated router. For these reasons, we will present an enhanced version of the protocol in the following section that operates without source routing, omits packet loss and attains expedited forwarding and protocol convergence.

\section{The Enhanced Tree Morphing Protocol}

\subsection{Overview}

A mobile multicast source (MS) away from home will transmit unencapsulated data to a group using its current CoA on the Internet layer, but its HoA on the application layer, which is carried in mobility extension headers defined in MIPv6. In extension to unicast routing, though, the multicast routing layer will be aware of the permanent HoA by maintaining $(C o A, H o A, G)$ address triples in router states.

When moving to a new point of attachment, the MS will alter its address from previous $\mathrm{CoA}(\mathrm{pCoA})$ to new $\mathrm{CoA}(\mathrm{nCoA})$ and eventually change from its previous Designated multicast Router (pDR) to a next Designated Router (nDR). Subsequent to handover, it will immediately initiate forwarding states on the route from $\mathrm{nDR}$ to pDR by submitting a regular unicast state update packet (cf. section 3.2) to pDR. As a result, the previous delivery tree will be elongated by $(n C o A, H o A, G)$ states as shown in figure 1(a). MS then continues to deliver data along this extended previous source tree. Delivery is done by including state update message headers in the first data packet(s). All routers along the previous delivery tree will thereby learn MS's new CoA and add appropriate forwarding states.

Routers participating in the ETM protocol will provide two additional functions: The State Injection Algorithm and the Extended Forwarding AlgoRITHM as shown below. State injection is triggered by the state update messages carried in a Hop-by-Hop option header. On each hop the new $(n C o A, H o A, G)$ state is implemented on the forwarding interfaces of the previous $(p C o A, H o A, G)$-state 


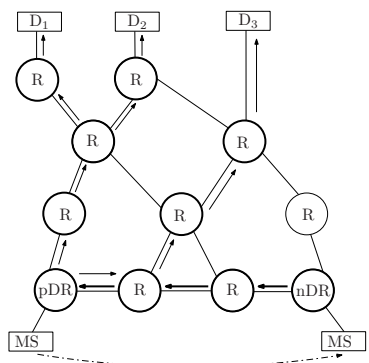

(a) Initial Tree with Elongation

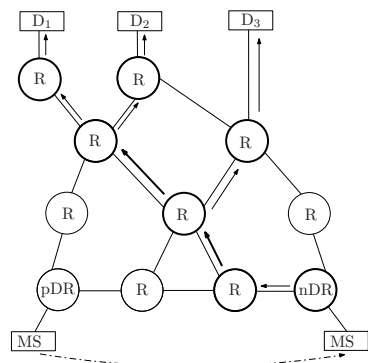

(b) Inter-Tree Morphing

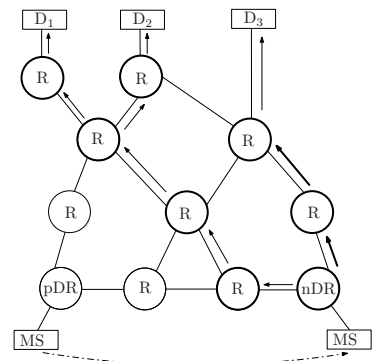

(c) Converged New Tree

Fig. 1 Tree Morphing States Neighboring to the Source with Receiver Domains $D_{i}$

tree. Previous states are kept only if the update packet was received on a topological incorrect interface. In detail this algorithm runs as follows:

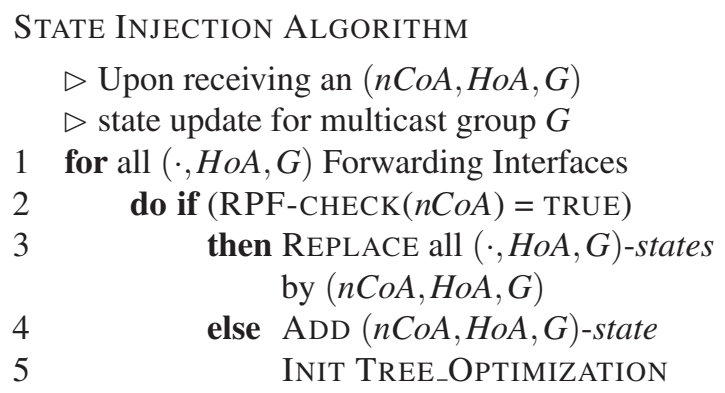

After the update has been processed, the packet is passed on according to the Extended Forwarding Algorithm explained below. At this stage, the delivery tree does not need to be optimal and packets may fail at standard RPF check. To prevent discarding, incoming packets need to be accepted from any interface, which is a topological member of the current or a previous distribution tree of $(\cdot, H o A, G)$ state. ${ }^{1}$ Therefore an extended forwarding, which accounts for all source address states $(\cdot, H o A, G)$, has to be applied until local tree optimization has completed. Packets thereby will be forwarded along an $(C o A, H o A, G)$ tree, provided they arrived at the topologically correct interface for this $C o A$.

Any router will observe suboptimal routes from packets arriving at a topological incorrect interface (w.r.t. the packet source address). As part of the algorithm it will then dynamically attempt to join to an optimal shortest path tree. When receiving a multicast packet for group $(\cdot, H o A, G)$ with source address $n C o A$ at the wrong interface, a router will immediately submit a join to $(n C o A, G)$. The underlying SSM routing protocol will initiate the construction of a shortest path source specific branch. The router will learn about its completion by $(n C o A, H o A, G)$ traffic arriving

${ }^{1}$ Further on we will denote "some state with group address $G$ and home address $H o A$ " by $(\cdot, H o A, G)$, whereas $(*, H o A, G)$ stands for all such states. 
at the correct interface and will then prune $(*, H o A, G)$ on all incoming interfaces corresponding to previous $C o A$ addresses. Fig. 1(b) visualizes such an intermediate morphing state.

A tree will be locally optimal, as soon as packets arrive at the topological correct interface. The details of this extended forwarding algorithm read:

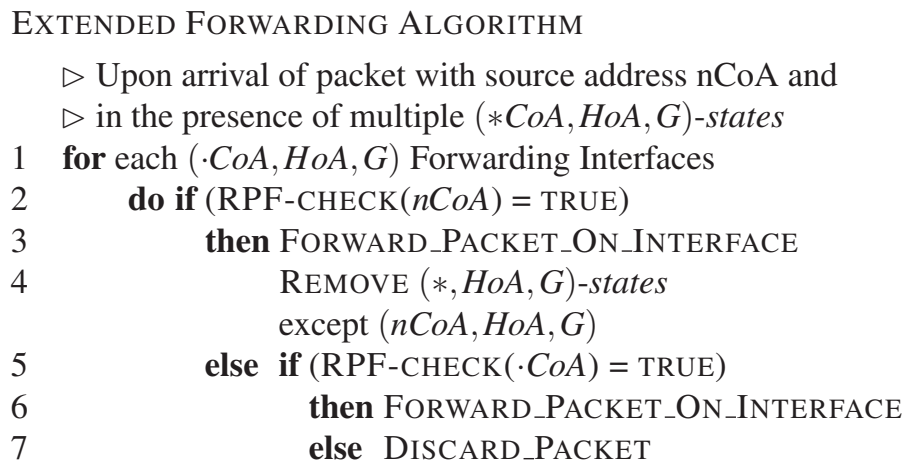

In applying this forwarding algorithm, the delivery tree thus will not only transport intermediate detouring packets, but will continuously degenerate branches dispensable due to optimization incidences. As soon as $(*, H o A, G)$ forwarding states have reduced to a single $(\cdot, H o A, G)$ entry, the router operations continue as defined by its standard multicast routing protocol without mobility extension.

Finally, state update packets will arrive at the receivers of the $(\cdot, H o A, G)$ SSM group. The mobile IPv6 stack will interpret the binding update and alter its multicast binding cache entry. Thereafter the standard destination option header is processed and data is transparently passed as $(H o A, G)$ to the transport layer.

\subsection{Packet Design}

The networking layer requires information of state changes, whenever a multicast source address changes after a Mobile IPv6 handover. This signaling is implemented on the network layer by inserting additional headers into the data packets and bears the common risks of a global redirect. The required information, group address, home address and care-of address, as well as proof of authentication can already be extracted of Binding Update messages sent by mobiles to correspondent end nodes subsequent to every unicast handover. The State Update Message needed for multicast can therefore be composed of several Mobile IPv6 headers and there is no need to define a new protocol from scratch. ETM messages can thus be processed transparently with regular, CGA authenticated [1] Binding Updates. Nevertheless they need to be interpreted by routers along the packet's path, which is achieved by a Router Alert Option inserted in a Hop-by-Hop Option Header [11]. This option is used to instruct routers to further inspect packet headers. 


\begin{tabular}{|c|c|c|c|c|c|c|}
\hline $\begin{array}{c}\text { IPv6 } \\
\text { Header }\end{array}$ & $\begin{array}{c}\text { Hop-by-Hop } \\
\text { Options } \\
\text { Header }\end{array}$ & $\begin{array}{c}\text { Dest. } \\
\text { Options } \\
\text { Header }\end{array}$ & \multicolumn{3}{|c|}{ Mobility Header } & \multirow{2}{*}{$\begin{array}{c}\text { Upper Layer } \\
\text { Header + Data } \\
\text { Data }\end{array}$} \\
\hline $\begin{array}{c}\text { Src: } \mathrm{CoA} \\
\text { Dst: G }\end{array}$ & $\begin{array}{c}\text { Router Alert } \\
\text { Option }\end{array}$ & $\begin{array}{l}\text { Home } \\
\text { Address } \\
\text { Option }\end{array}$ & \begin{tabular}{|c|} 
Binding \\
Update \\
Message
\end{tabular} & $\begin{array}{l}\text { CGA } \\
\text { Param. } \\
\text { Option }\end{array}$ & $\begin{array}{c}\text { CGA } \\
\text { Signature } \\
\text { Option }\end{array}$ & \\
\hline
\end{tabular}

Fig. 2 ETM IPv6 Header Sequence for Authenticated State Updates of Mobile Multicast Sources

The complete signaling is built by chaining the IPv6 extension headers as to be sent in the initial unicast state update message as well as for piggy-backing with the first data packet(s). Figure 2 shows the combined packet format used after source handover. This mechanism generalizes to an integrated, secure update protocol for mobile multicast sources named 'AuthoCast' in forthcoming work.

\subsection{ETM Finite State Machine}

The finite state machine of the ETM protocol is derived of the PIM-SSM [4] state machine. The states No Info (NI), Join (J) and Prune Pending (PP) interact as in standard PIM-SSM. The Join state is augmented by an ETM state, which represents the ETM router conditions during mobility management and prior to protocol convergence. Its characteristic lies in a state splitting initiated from state update messages, which in the event of rapid movement may be received multiple times prior to convergence. This is realized via a state counter and allows for a joined treatment of the correlated $(\cdot, H o A, G)$ stated within routers. The FSM has been used to verify the protocol formally.

\section{Protocol Evaluation}

To evaluate the protocol behavior, we implemented the corresponding functions of routers, sources and receivers within the network simulator platform OMNeT++ 3.3 [19] on top of the IPv6Suite, which is based on the INET framework and already realizes MIPv6 protocol operations. We performed a stochastic discrete event simulation, firstly choosing artificial topologies, which explore the attainable relative network geometry, and several real-world measurements. In detail, the simulation proceeds as follows: The mobile source continuously submits (numbered and timestamped) packets at a constant bit rate of $15 \mathrm{~ms}$, while performing a handover from one WLAN access point to another. Access points are directly connected to the designated routers. Link delays in our setting have been homogenously chosen to be $10 \mathrm{~ms}$.

Our analysis covers packet delay, loss and convergence times. Measurements have been performed with the help of a monitoring function at routers and receivers, which accounts for the maximal delay stretch, i.e., the ratio taken of the slowest 
packet, delivered during handoff, over the optimal time, a surveillance of packet delivery at the receivers, and a state observation for protocol convergence. It should be noted that there are two relevant convergence times. Prior to a full protocol convergence, i.e., a leave of the ETM state in all routers, packets may be already delivered on optimal paths. This convergence to optimal forwarding has been monitored separately at the receivers.

\subsection{Analyzing the Network Topology Space}

For a systematic approach to routing analysis, we first proceed in artificially exploring the topology space, i.e., the relative positions of the relevant network entities. The latter are given by the designated routers and the first intersection point (X) of previous and new multicast tree. The degrees of freedom, which only depend on distance ratios, are covered by the two networks displayed in figure 3 .

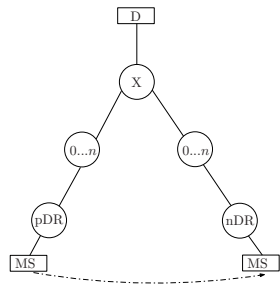

(a) Net 1

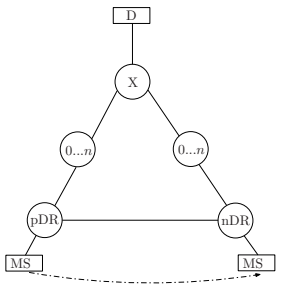

(b) Net 2

Fig. 3 Test Networks Covering the Relative Routing Topology

The simulation results for the two test networks as functions of intermediate router hops *DR-to-X chosen between 0 and 30 are given in figure 4 . As a striking outcome, test net 1 always delivers optimal forwarding without additional delays. This is due to the immediate discovery of the intersection router $\mathrm{X}$ by the EXTENDED FORWARDING ALGORITHM, which leads to packet delivery on the shortest path directly following a handover. Test net 2 admits similar performance values, but requires a re-routing, whenever the path lengths pDR-X equals nDR-X. This is reflected by a non-normal delay stretch and non-zero convergence time to optimal forwarding along a diagonal of the graph. Note that the characteristic of the delay stretch derives from the changing optimal forwarding times, while the absolute delay excess stems from one additional hop, i.e., nDR-X. In contrast, routing convergence to final states is non-negligible in most cases. Routers along the path from $\mathrm{nDR}$ to $\mathrm{pDR}$ will change into TM state, the routers on the previous tree, i.e., along X-pDR, will remain therein until a regular PIM prune is received. For test net 2 forwarding states will be simply overwritten, when the distribution tree remains unaltered, i.e., $\operatorname{dist}(n D R, X) \neq \operatorname{dist}(p D R, X)$. A PIM prune towards $\mathrm{pDR}$ is 


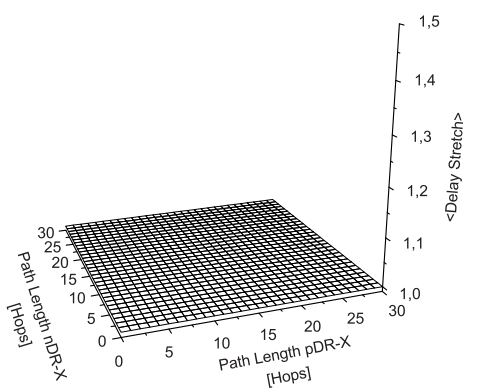

(a) Delay Stretch Net 1

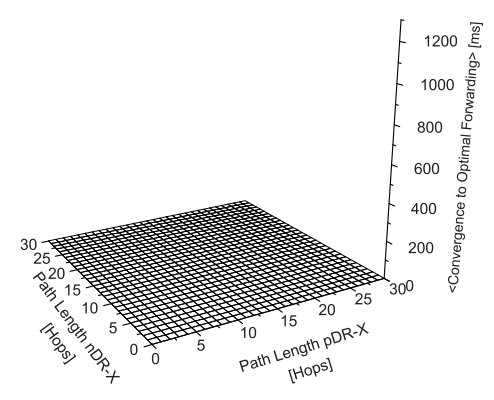

(c) Optimal Forwarding Net 1

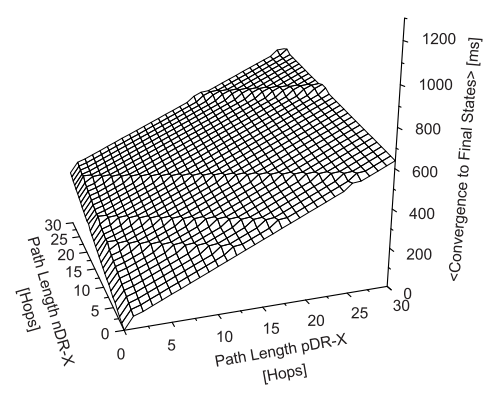

(e) Final Convergence Net 1

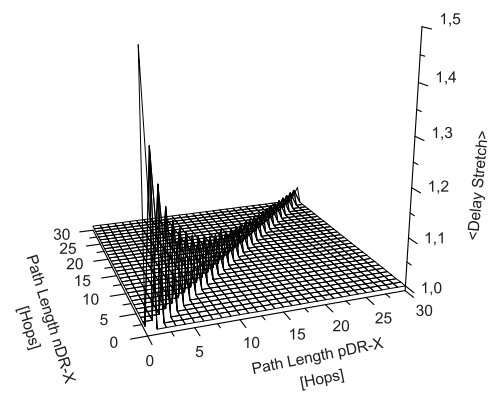

(b) Delay Stretch Net 2

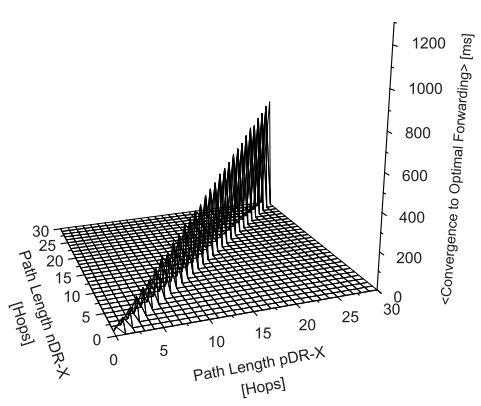

(d) Optimal Forwarding Net 2

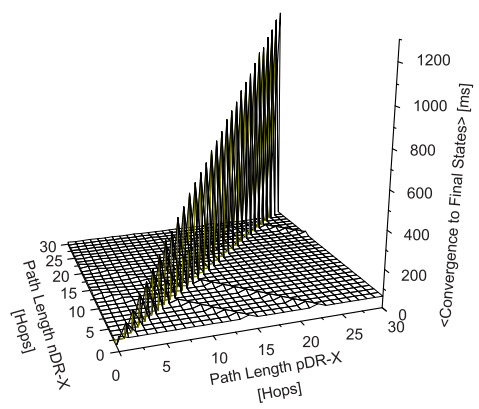

(f) Final Convergence Net 2

Fig. 4 Performance Results for the Test Networks

required for $\operatorname{dist}(n D R, X)<\operatorname{dist}(p D R, X)$, while for $\operatorname{dist}(n D R, X)=\operatorname{dist}(p D R, X)$ the tree changes with the result of join and prune operations along the entire paths. No packet loss occurs in any case, why corresponding graphs are omitted. 


\subsection{Simulations Based on Real-World Topologies}

To approximate realistic scenarios, further protocol evaluation has been performed on the basis of real-world topologies. Network data must be considered critical, as key characteristics of multicast routing are dominated by the topology in large networks. We chose the ATT core network [6] as a large (154 core nodes), densely meshed single provider example. For multiple provider Internet data we extracted a sub-samples of 154 and 1.540 core nodes from the "SCAN + Lucent" map [5] project, further on denoted as "Internet" topology.

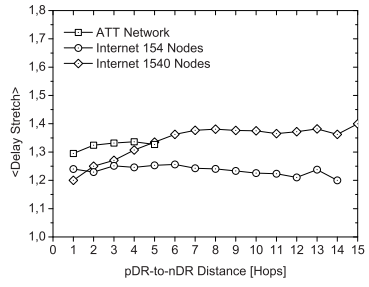

(a) Delay Stretch

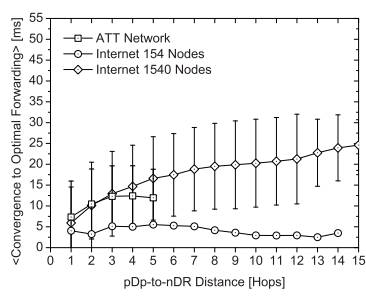

(b) Optimal Forwarding

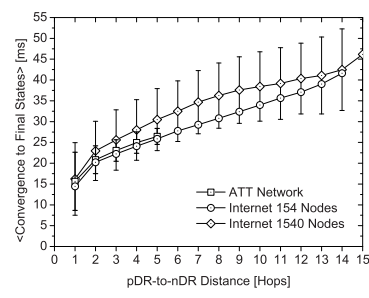

(c) Final Convergence

Fig. 5 Performance Results for Real-World Topologies (Error Bars show Standard Deviation)

Mean performance results are shown in figure 5 as functions of pDR-nDR distance. ${ }^{2}$ Packet loss, which is only possible, when initial routing is guided along uneven triangles, did occur at only a couple events. Losses remain invisible in distributions, why the corresponding graphs are again not shown. The initial delay stretches for the topologies vary at an excess rate of about 20 to $35 \%$, almost independent of access router distance. The convergence times to optimal forwarding remain bound by about $20 \mathrm{~ms}$. At our probe packet rate, the second datagram thus travels an optimal path. Final convergence times clearly represent routing complexity as a function of mobility 'step size' and of topological diversity at comparably low values. Combinedly, the results indicate that the extremal values obtained for the artificial topologies equal out nicely when mixing in realistic topological settings.

\section{Conclusions and Outlook}

In this article the Enhanced Tree Morphing protocol was introduced and extensively evaluated. ETM is an optimized improvement of the previously developed Tree Morphing. ETM abandons the source routing of packets used in TM and separates control packets from the data in its initial tree elongation phase. These op-

\footnotetext{
2 The access router distance, the mobility 'step size' in a figurative sense, can be regarded as a measure of complexity inherent to the problem [15]. Values range up to 15 in the Internet topology samples, while the maximum router distance within the ATT network is 5 .
} 
timizations significantly advanced the protocol performance, as could be derived from a systematic protocol evaluation. In future work we will focus on analyzing the protocol robustness against network disruptions from strong bursts and packet overflow, as well as on the case of rapid movement of the mobile source. AuthoCast, an integrated secure state update protocol for mobile multicast will be forthcoming.

\section{References}

1. Arkko, J., et al.: Enhanced Route Optimization for Mobile IPv6. RFC 4866, IETF (2007)

2. Chang, R.S., Yen, Y.S.: A Multicast Routing Protocol with Dynamic Tree Adjustment for Mobile IPv6. Journ. Information Science and Engineering 20, 1109-1124 (2004)

3. Deering, S.E.: Host Extensions for IP Multicasting. RFC 1112, IETF (1989)

4. Fenner, B., Handley, M., Holbrook, H., Kouvelas, I.: Protocol Independent Multicast - Sparse Mode (PIM-SM): Protocol Specification (Revised). RFC 4601, IETF (2006)

5. Govindan, R., Tangmunarunkit, H.: Heuristics for internet map discovery. In: Proceedings IEEE INFOCOM 2000, vol. 3, pp. 1371-1380. IEEE Press, Piscataway, NJ, USA (2000).

6. Heckmann, O., Piringer, M., Schmitt, J., Steinmetz, R.: On Realistic Network Topologies for Simulation. In: MoMeTools '03, pp. 28-32. ACM Press, NY, USA (2003)

7. Holbrook, H., Cain, B.: Source-Specific Multicast for IP. RFC 4607, IETF (2006)

8. Jelger, C., Noel, T.: Supporting Mobile SSM sources for IPv6 (MSSMSv6). Internet Draft work in progress (expired) 00, individual (2002)

9. Johnson, D.B., Perkins, C., Arkko, J.: Mobility Support in IPv6. RFC 3775, IETF (2004)

10. O'Neill, A.: Mobility Management and IP Multicast. Internet Draft - work in progress (expired) 01, IETF (2002)

11. Partridge, C., Jackson, A.: IPv6 Router Alert Option. RFC 2711, IETF (1999)

12. Romdhani, I., Bettahar, H., Bouabdallah, A.: Transparent handover for mobile multicast sources. In: P. Lorenz, P. Dini (eds.) Proceedings of the IEEE ICN'06. IEEE Press (2006)

13. Rosenberg, J., Schulzrinne, H., Camarillo, G., Johnston, A., Peterson, J., Sparks, R., Handley, M., Schooler, E.: SIP: Session Initiation Protocol. RFC 3261, IETF (2002)

14. Schmidt, T.C., Wählisch, M.: Extending SSM to MIPv6 - Problems, Solutions and Improvements. Computational Methods in Science and Technology 11(2), 147-152 (2005).

15. Schmidt, T.C., Wählisch, M.: Morphing Distribution Trees - On the Evolution of Multicast States under Mobility and an Adaptive Routing Scheme for Mobile SSM Sources. Telecommunication Systems 33(1-3), 131-154 (2006).

16. Schmidt, T.C., Wählisch, M.: Group Conference Management with SIP. In: S. Ahson, M. Ilyas (eds.) SIP Handbook: Services, Technologies, and Security. CRC Press (2008).

17. Schmidt, T.C., Wählisch, M.: Multicast Mobility in MIPv6: Problem Statement and Brief Survey. IRTF Internet Draft - work in progress 03, MobOpts (2008).

18. Thaler, D.: Supporting Mobile SSM Sources for IPv6. Proceedings of ietf meeting (2001).

19. Varga, A.: The OMNeT++ discrete event simulation system. http://www.omnetpp.org (2007)

20. Wählisch, M., Schmidt, T.C.: Exploring the Routing Complexity of Mobile Multicast - A Semi-empirical Study. In: CoNEXT '07 Proceedings. ACM, NY (2007).

21. Xylomenos, G., Polyzos, G.C.: IP Multicast for Mobile Hosts. IEEE Comm. Mag. 35(1), 54-58 (1997) 